\title{
Estudio de supervivencia de pacientes menores de 18 años de edad con diagnóstico de cáncer atendidos en el Hospital Universitario San Ignacio-Centro Javeriano de Oncología entre octubre de 2010 y marzo de 2016
}

Survival Study of Pediatric Patients Diagnosed with Cancer Treated at the Hospital Universitario San Ignacio-Centro Javeriano de Oncología between October 2010 and March 2016

\footnotetext{
Paula Carolina Guzmán Cruz

Hospital Universitario San Ignacio, Colombia

Luis Enrigue FARIAs Curtidor ${ }^{\mathrm{a}}$

Pontificia Universidad Javeriana, Colombia

Helena María Jiménez Villabona

Pontificia Universidad Javeriana, Colombia

Patricia Maldonado Riveros

Pontificia Universidad Javeriana, Colombia

Iliana del Carmen de los Reyes

VALENCIA

Hospital Universitario San Ignacio, Colombia
}

\footnotetext{
${ }^{a}$ Autor de correspondencia. Correo electrónico: luisefcdoc@gmail.com

Cómo citar: Guzmán Cruz PC, Farías Curtidor LE, Jiménez Villabona HM, Maldonado Riveros P, De los Reyes Valencia IC. Estudio de supervivencia de pacientes menores de 18 años de edad con diagnóstico de cáncer atendidos en el Hospital Universitario San Ignacio-Centro Javeriano de Oncología entre octubre de 2010 y marzo de 2016. Univ Med. 2017; 58(4): 1-7. doi: http://dx.doi.org/10.11144/Javeriana. umed58 -4.espm
}

\section{RESUMEN}

Introducción: El cáncer infantil ocasiona importante mortalidad. El objetivo es caracterizar y determinar la sobrevida de un centro especializado. Métodos: Análisis de supervivencia en menores de 18 años de edad, diagnosticados con cáncer del 01/10/2010 al 31/03/2016. Se analizaron variables sociodemográficas (edad, sexo, seguridad social y zona de residencia) y clínicas (tipo de cáncer y categorización del riesgo). Se utilizó el método de Kaplan-Meier y Log-Rank Test. Resultados: 135 pacientes, con una edad promedio 7,4 años (DE 4,84), la mayoría de sexo masculino y pertenecientes al régimen contributivo. Las neoplasias más frecuentes fueron leucemias y tumores de sistema nervioso central, predominancia de alto riesgo. La supervivencia global fue del $75 \%$ y la supervivencia libre de evento fue del $60 \%$. Los tumores sólidos y de alto riesgo presentaron mayor mortalidad. Conclusiones: La supervivencia global a 5 años es similar a los datos internacionales. La mayoría de los tumores sólidos eran de alto riesgo, probablemente asociados a mayor tiempo de evolución.

Palabras clave

neoplasias; pediatría; supervivencia; progresión; recaída. 
Introduction: Childhood cancer is a major cause of mortality. The aim is to characterize the population and determine the survival of child population in a reference center. Methods: Survival analysis patients younger than 18 years diagnosed with cancer $01 / 10 / 2010$ to $31 / 03 / 2016$. Socio demographic variables (age, sex, social security and area of residence) and clinical (type of cancer and risk categorization) were analyzed. The Kaplan-Meier and Log Rank Test was used. Results: 135 patients were evaluated, the average age at diagnosis was 7,4 years (SD 4,84), mostly male, contributory scheme and urban residence. The most frequent neoplasms were leukemias and central nervous system tumors and most were high-risk. Overall survival was $75 \%$ and event-free survival of $60 \%$. Solid and high-risk tumors had a higher mortality. Conclusions: The overall 5-year survival is similar to international data. Most solid tumors were high risk probably associated with a longer history.

Keywords

neoplasm; pediatrics; survival; disease progression; recurrence.

\section{Introducción}

El cáncer es una enfermedad que amenaza la vida, y aunque es poco frecuente en la población infantil, es la segunda causa de mortalidad en dicha población después de los accidentes [1,2]. La incidencia anual para todos los tumores malignos es de 12,45 por cada 100.000 niños menores de 15 años de edad [1].

A pesar del avance en los tratamientos y su mayor efectividad, existe una brecha importante en las tasas de supervivencia, dependiendo del contexto socioeconómico y demográfico en el que se encuentran los pacientes, pues los niños en países en vías de desarrollo tienen una supervivencia 10-20\% menor, comparada con la de niños en sus mismas circunstancias de salud, pero que viven en un país desarrollado, con tasas de supervivencias reportadas en Colombia de solo el $43 \%$, para el 2013 [1,3]. Las causas de estas diferencias se deben a diagnóstico tardío, acceso limitado al tratamiento, abandono al tratamiento y recurrencia de la enfermedad [4]; además de lo anterior hay una gran variabilidad en los modelos de atención en cada una de las instituciones donde se manejan estos pacientes.

Al diferenciar por tipo de neoplasia, se estima que la leucemia representa un tercio de todos los tipos de cáncer, esto es, la mayoría de los casos están representados por las leucemias linfocíticas agudas. Las neoplasias del sistema nervioso central son el segundo tipo de cáncer más común (25\%), seguido de los sarcomas de tejidos blandos (7\%), neuroblastoma (6\%), tumores renales $(5 \%)$ y linfomas de Hodgkin y no Hodgkin (4\%, cada uno) [5].

En Colombia, el cáncer en niños no representa más del $3 \%$ de los casos nuevos del país, pero sí tiene altas tasas de mortalidad, especialmente por leucemias agudas pediátricas. Según la Liga Colombiana de Lucha contra el Cáncer, esta enfermedad resulta ser la segunda causa de muerte infantil en el país [1].

Según el Instituto Nacional de Cancerología, la mortalidad para todo tipo de cáncer en menores de 14 años en Colombia en el 2011 era de 4,1/100.000 habitantes para hombres y de 3,8/100.000 habitantes para mujeres. Para leucemias era de 2,3/100.000 habitantes para hombres y de 1,8/100.000 habitantes para mujeres, respectivamente [6]. Sin embargo, los datos respecto a los pacientes oncológicos pediátricos son limitados, con reportes que mencionan tasas de supervivencias globales menores del $50 \%$ [3]. Por lo anterior, se considera pertinente obtener información epidemiológica enfocada en este tipo de patología en la población infantil.

El propósito principal del estudio es caracterizar la distribución porcentual y de sobrevida de población infantil con neoplasias atendida en el Hospital Universitario San Ignacio-Centro Javeriano de Oncología, con un modelo de atención que incluye un servicio de pediatría con una unidad de oncología pediátrica dentro de un hospital general universitario.

\section{Materiales y métodos}

Se realizó un análisis de supervivencia que incluyó a 135 pacientes, seleccionados bajo los siguientes criterios de inclusión: todos menores de 18 años de edad, atendidos en el Hospital Universitario San Ignacio-Centro Javeriano de Oncología, con diagnóstico confirmado de cáncer, en el periodo 1 de octubre del 2010-31 
de marzo del 2016. Se excluyeron los pacientes tratados en otros centros.

Se creó en Excel ${ }^{\circledR}$ una base de datos con las variables de interés; además, dos evaluadores revisaron las historias clínicas, con el fin de minimizar los errores de transcripción de la historia clínica a la base de datos.

En la base de datos se incluyeron variables sociodemográficas (edad en el momento del diagnóstico, sexo, tipo de seguridad social y zona de residencia) y clínicas (tipo de cáncer y categorización del riesgo según el protocolo del tratamiento utilizado). Para los análisis estadísticos se utilizó el software Stata 10.

Para realizar el cálculo de supervivencia global y supervivencia libre de evento se utilizó el método de Kaplan-Meier y para comparar los grupos el Log-rank Test, se consideraron eventos: progresión, recaída, abandono o muerte; en caso de presencia de recaída, progresión o abandono se consideró desenlace muerte.

\section{Resultados}

\section{Caracterización de la población}

Se caracterizaron las variables sociodemográficas de los 135 pacientes que cumplieron con los criterios para ser incluidos en el estudio. La edad promedio al diagnóstico fue de 7,4 años, con una edad mínima de 3 meses y una máxima de 16 años. La desviación estándar para la edad al diagnóstico fue de 4,84 años. El sexo predominante fue masculino, con un $61,4 \%$; el tipo de seguridad social fue el contributivo, con $73,3 \%$, y la zona de residencia más frecuente fue la urbana con un 90,3\% del total (tabla 1).
Tabla 1. Características sociodemográficas $(n=135)$

\begin{tabular}{|l|l|r|}
\hline \multicolumn{2}{|c|}{ Edad } & \multicolumn{2}{|l|}{ Promedio 7,4 años; DE: 4,84 años } \\
\hline \multirow{2}{*}{ Sexo } & 1. Masculino & n ( \%) \\
\cline { 2 - 3 } & 2. Femenino & $83(61,4)$ \\
\hline \multirow{2}{*}{$\begin{array}{l}\text { Seguridad } \\
\text { social }\end{array}$} & 1. Contributivo & $52(38,5)$ \\
\cline { 2 - 3 } & 2. Subsidiado & 39 $(73,3)$ \\
\cline { 2 - 3 } $\begin{array}{l}\text { Zona de } \\
\text { residencia }\end{array}$ & 3. Vinculado & $1(25,9)$ \\
\cline { 2 - 3 } & 2. Urbano & $122(90,37)$ \\
\hline
\end{tabular}

En cuanto al tipo de cáncer, los más frecuentes fueron leucemias y tumores de sistema nervioso central, con un $25,9 \%$ y un $26,6 \%$, respectivamente; cerca del $70 \%$ de los tumores fueron de alto riesgo (tabla 2).

Tabla 2. Características clínicas

\begin{tabular}{|l|l|r|}
\hline \multirow{4}{*}{ Tipo de cáncer } & 1. Leucemias & $35(25,9 \%)$ \\
\cline { 2 - 3 } & 2. Linfomas & $14(10,3 \%)$ \\
\cline { 2 - 3 } & 3. Tumores de sistema nervioso central & $36(26,6 \%)$ \\
\cline { 2 - 3 } & 4. Tumores de hueso y tejidos blandos & $25(18,5 \%)$ \\
\cline { 2 - 3 } & 5. Otros tumores solidos & $25(18,5 \%)$ \\
\hline \multirow{2}{*}{ Estadificación inicial } & 1. Bajo riesgo & $41(30,3 \%)$ \\
\cline { 2 - 3 } & 2. Alto riesgo & $44(69,6 \%)$ \\
\hline \multirow{2}{*}{$\begin{array}{l}\text { Tiempo promedio de } \\
\text { seguimiento en días }\end{array}$} & 1. Hematolinfoides & 706,4 días $(23,5$ meses $)$ \\
\cline { 2 - 3 } & 2. Sólidos & 626,6 días $(20,8$ meses $)$ \\
\hline
\end{tabular}

\section{Supervivencia global}

En la figura 1 se observa la supervivencia global de los 135 pacientes evaluados. El 74,8\% ( $\mathrm{n}=$ 101) estaban vivos en el momento de completar el seguimiento en el estudio (18 de agosto de 2016); mientras que el $25,2 \%(\mathrm{n}=34)$ había fallecido.

Figura 1. Supervivencia global

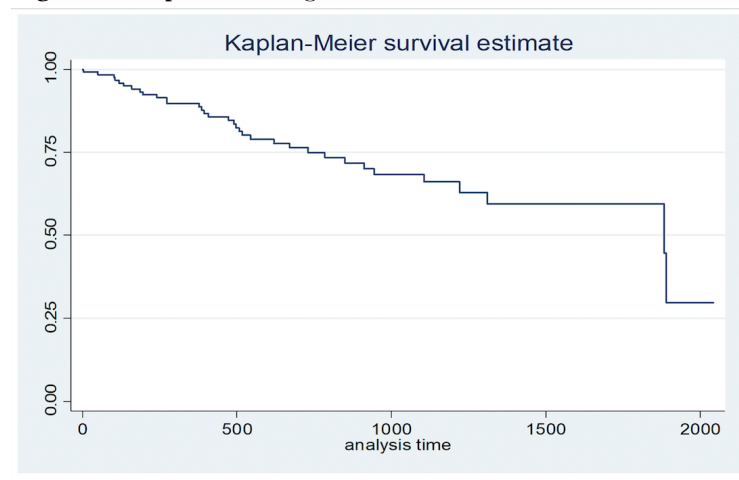

Los pacientes con último estado vital registrado como vivo tuvieron un promedio de seguimiento de 708 días (con un mínimo de 5 
días y un máximo de 2042 días). Los pacientes fallecidos tuvieron un promedio de días de seguimiento de 569 (con un mínimo de 2 días y un máximo de 1890 días). El tiempo promedio de seguimiento fue de 706 días para tumores hematolinfoides y de 626 días para tumores sólidos. La mediana de supervivencia global fue de 1880 días, aproximadamente.

Supervivencia libre de evento

Al realizar el análisis de supervivencia libre de evento en los 135 pacientes incluidos en el estudio, se evidenció que aproximadamente a los 950 días de seguimiento, el $60 \%$ de los pacientes no había presentado ninguno de los eventos evaluados en el estudio (muerte, progresión, recaída o abandono). No se calculó la mediana de supervivencia libre de evento, teniendo en cuenta que para el final del tiempo de seguimiento menos del $50 \%$ de los pacientes presentaron alguno de los eventos planteados (figura 2).

Figura 2. Supervivencia libre de evento

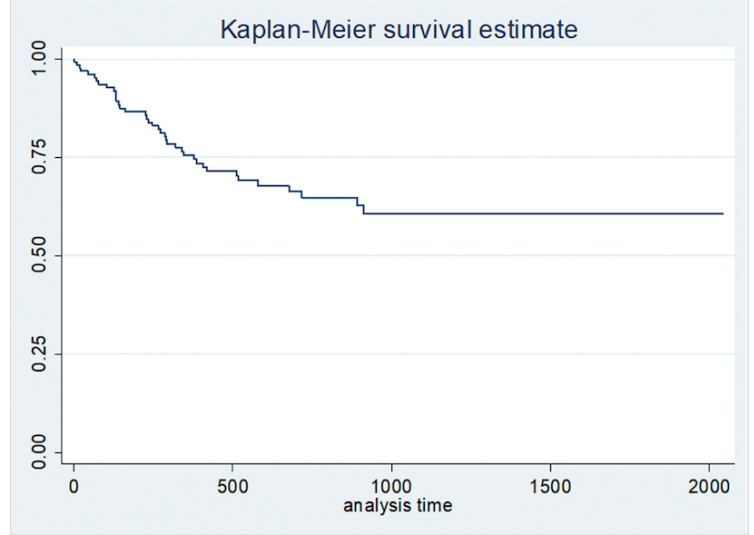

Supervivencia libre de evento (discriminando tipo de evento)

Al evaluar la supervivencia libre de evento, se identificaron $95(70,3 \%)$ pacientes que no presentaron ninguno de los eventos que se iban a evaluar durante el tiempo de seguimiento. $\mathrm{Al}$ discriminar por tipo de evento, encontramos 23 (17\%) pacientes con progresión y $10(7,4 \%)$ con recaída; adicionalmente, 6 (5,3\%) de los pacientes fallecieron sin presentar progresión o recaída previos. La mediana de supervivencia para progresión fue de 226 días, y para recaída, 320 días (figura 3).

Figura 3. Supervivencia libre de evento (discriminada por tipo de evento)

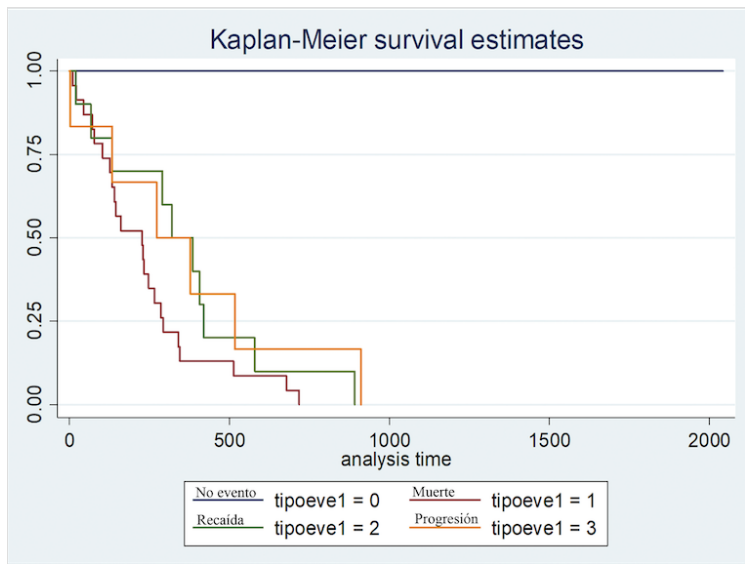

Supervivencia global por subgrupo de cáncer: sólidos vs. hematolinfoides

En el análisis de supervivencia por tipos de cáncer (tumores hematolinfoides comparados con tumores sólidos) hubo un mayor porcentaje de mortalidad en el grupo de tumores sólidos (33\%) comparado con una mortalidad del 10 $\%$ de tumores hematolinfoides. La mediana de supervivencia para los pacientes con tumores sólidos fue de 1200 días, y para los hematolinfoides no se calculó teniendo en cuenta que la mayoría de pacientes estaban vivos hasta el momento de fin del seguimiento del presente estudio (figura 4). Se realizó el análisis de Log Rank Test en el que se encontró una diferencia significativa entre los subgrupos ( $\mathrm{p}$ menor de 0,001). 
Figura 4. Supervivencia global por tipo de cáncer (sólido vs. hematolinfoide)

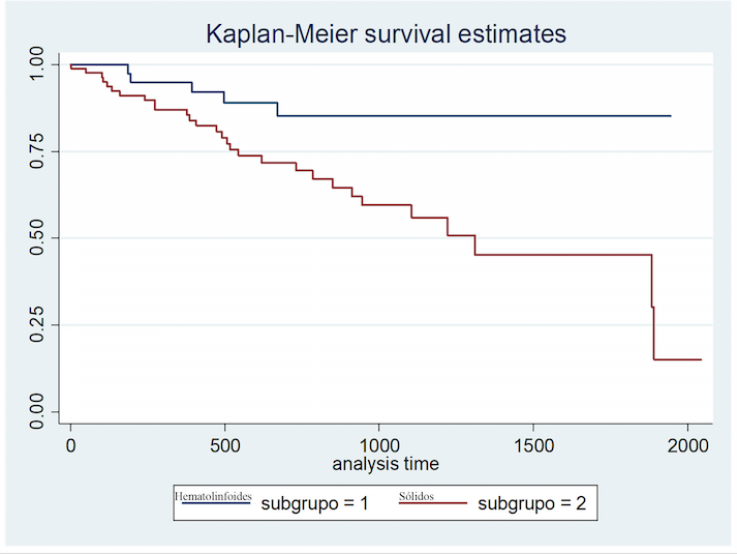

Supervivencia libre de evento por tipo de cáncer: sólidos vs. hematolinfoides

Solo el $20 \%$ de los pacientes con tumores hematolinfoides presentó algún evento, comparado con el $50 \%$ en los tumores sólidos. Se realizó el análisis de Log Rank Test en el que se encontró una diferencia significativa entre los subgrupos (p menor de 0,001). Véase la figura 5.

Figura 5. Supervivencia libre de evento por tipo de cáncer (hematolinfoide vs. sólido)

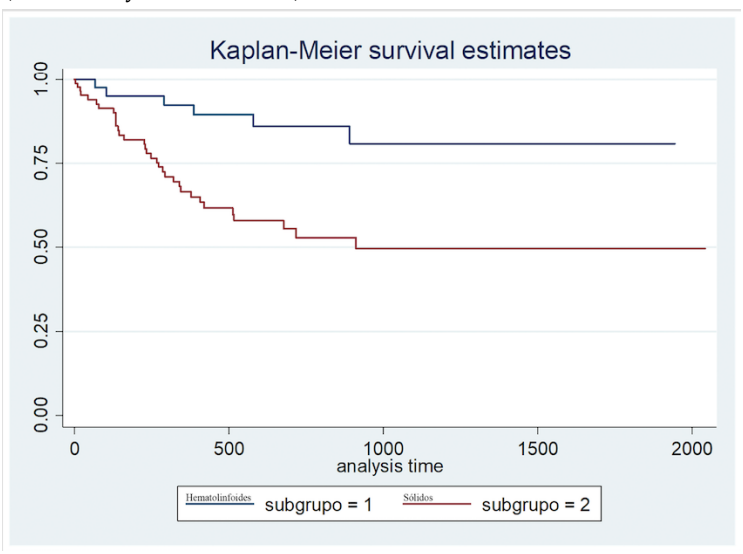

Supervivencia global y libre de evento por estadificación inicial

La supervivencia global y libre de evento de subgrupos diferenciados por estadificación inicial de riesgo demuestra que la supervivencia para los pacientes de alto riesgo es menor comparada con pacientes de bajo riesgo (figuras 6 y 7). Se realizó el análisis de Log Rank Test que se encontró una diferencia significativa entre los subgrupos ( $\mathrm{p}$ menor de 0,001).

Figura 6. Supervivencia global diferenciada por estadificación inicial (alto riesgo vs. bajo riesgo)

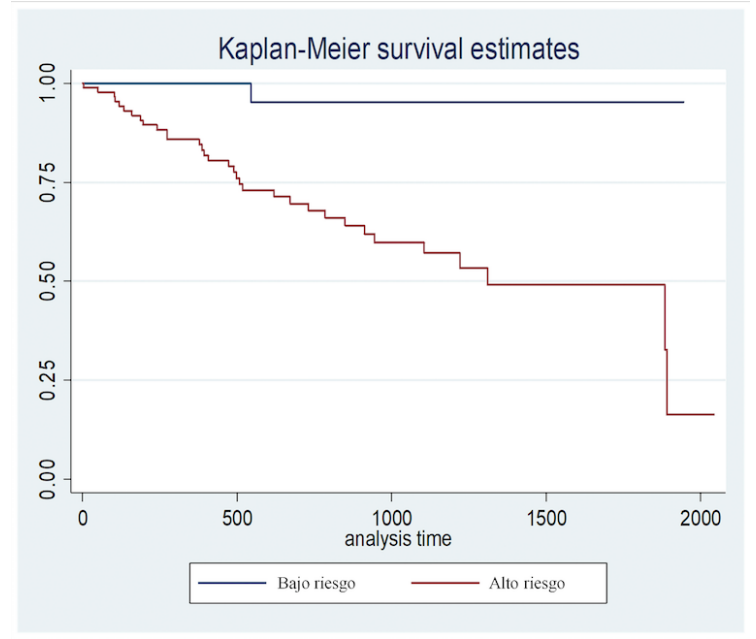

Figura 7. Supervivencia libre de evento por estadificación inicial: bajo riesgo ws. alto riesgo

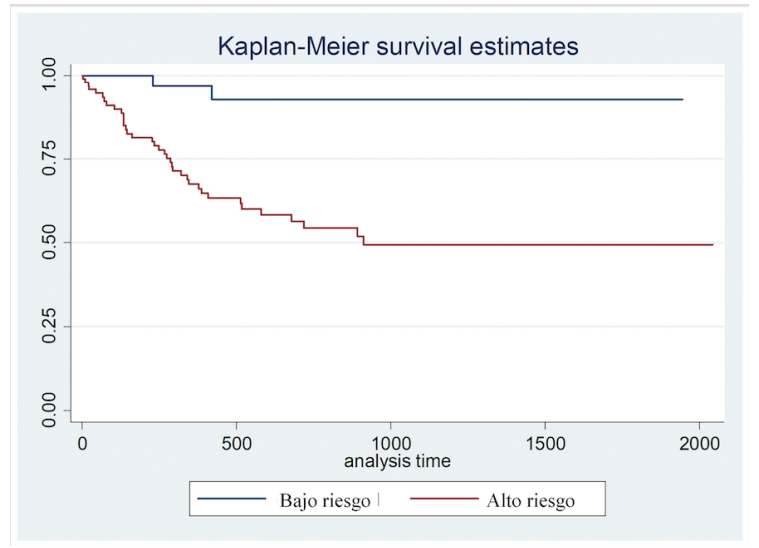

\section{Discusión y conclusiones}

Los resultados de nuestro estudio en cuanto a frecuencia de tipo de cáncer concuerdan con las estadísticas internacionales [5], teniendo en cuenta que las neoplasias más frecuentes fueron leucemias y tumores de sistema nervioso central.

En cuanto a supervivencia global a 5 años, el $75 \%$ de los pacientes estaba vivo al final de nuestro estudio, que tuvo un seguimiento 
máximo de 2042 días (5,5 años). Esta cifra se correlaciona con datos del Instituto Nacional de Cáncer de Estados Unidos [7].

Los tumores sólidos tuvieron una mayor tasa de mortalidad, comparada con los hematolinfoides, dato que se correlacionan con la literatura internacional [8,9]. En comparación con el estudio previo de supervivencia de pacientes menores de 18 años diagnosticados con cáncer entre 2007 y 2010 y atendidos en el Centro Javeriano de Oncología [10], no hay diferencia en la supervivencia global y en nuestro estudio no se encontraron abandonos.

Aunque los datos en nuestro país varían dependiendo de cada institución y zona geográfica, la sobrevida global en Colombia sigue siendo baja, comparada con estándares internacionales [2]. En la institución evaluada se encontraron datos comparables con sobrevidas internacionales, y es de resaltar que no hubo ningún abandono, por lo que consideramos exitoso el modelo de atención de una unidad de oncología pediátrica dentro de un hospital general universitario.

Los datos del presente estudio solo son aplicables a la institución estudiada. Llama la atención que la mayoría de los pacientes con tumores sólidos fueron de alto riesgo, lo que puede sugerir un tiempo mayor a lo esperado entre el inicio de los síntomas y el diagnóstico, por lo que se requieren estudios dirigidos a profundizar en este tema. Adicionalmente, el tamaño de la muestra y el seguimiento es reducido.

\section{Agradecimientos}

A Martín Alonso Rondón Sepúlveda, magíster en Bioestadística de la Universidad de Santiago de Chile y estadístico del Departamento de Epidemiología y Estadística de la Pontificia Universidad Javeriana.

\section{Referencias}

1. Instituto Nacional de Salud. Protocolo de vigilancia en salud pública: cancer infantil [Internet]. 2014 [citado 2016 jun 20]. Disponible en http://www.ins.gov.co/lineas-de-accion/ Subdireccion-Vigilancia/sivigila/Protocolos \%20SIVIGILA/PRO\%20Cancer\%20Infan til.pdf

2. Organización Mundial de la Salud, Organización Panamericana de la Salud. AIEPI: Diagnóstico temprano del cáncer en la niñez [internet]. 2014 [citado 2016 jun 21]. Disponible en: http://www.iccp-portal.org/sites/default /files/resources/Manual\%20AIEPI_ESP.pdf

3. Ministerio de Salud de Colombia. Guía de práctica clínica para la detección oportuna, diagnóstico y seguimiento de leucemia linfoide aguda y leucemia mieloide aguda en niños, niñas y adolescentes [internet]. 2013 [citado 2016 jun 21]. Disponible en: https://www.minsalud.gov.co/sites/rid/ Lists/BibliotecaDigital/RIDE/INEC/IETS/ GPC_Prof_Sal_Leucemia.pdf

4. Hunger SP, Mullighan CG. Acute lymphoblastic leukemia in children. $\mathrm{N}$ Engl J Med. 2015;373:1541-52.

5. Howlader N, Noone AM, Krapcho M, et al. SEER cancer statistics review, 1975-2009 [internet]. National Cancer Institute. Bethesda, MD, 2012 [citado 2016 jun 19]. Disponible en http://seer.cancer.g ov/archive/csr/1975_2009_pops09/

6. Instituto Nacional de Cancerología. Magnitud de cancer en el país: mortalidad nacional por tipo de cancer y grupo de edad [internet]. [Citado 2016 jun 28]. Disponible en: http://www.cancer.gov.co/c ancer_en_cifras

7. Instituto Nacional del Cancer. Cánceres infantiles [internet]. 2015 [citado 2016 jun 23]. Disponible en: https://www.cancer.gov/espanol/tipos/i nfantil/hoja-informativa-ninos-adolescente $\mathrm{s}$

8. Warren KE. Diffuse intrinsic pontine glioma: poised for progress. Front Oncol. 2012;2:205. doi: 10.3389/fonc.2012.00205 
9. Ward E, DeSantis C, Robbins A, Kohler B JA. Childhood and adolescent cancer statistics, 2014. Cancer J Clin. 2014;64(2):83-103.

10. Manrique M, Narváez CE, Guzmán PC, Pedraza M. Estudio de supervivencia en pacientes pediátricos con cáncer atendidos en el centro javeriano de oncología. Univ Med. 2012;53(2):120-5. 